\title{
Intencionalidades y resistencias en el aprendizaje del inglés: referentes para diseñar estrategias didácticas efectivas
}

\author{
Intentionalities and resistances in English language learning: \\ References to design effective didactic strategies \\ Berenice Jaime Romero \\ Willelmira Castillejos López \\ Álvaro Reyes Toxqui
}

\begin{abstract}
RESUMEN
El propósito de este artículo es evidenciar la trascendencia de las intencionalidades y resistencias que se construyen en el entorno escolar como referentes para diseñar estrategias didácticas efectivas para el aprendizaje del inglés como lengua extranjera. Los postulados vertidos se basan en la integración de un modelo teórico de comprensión de la realidad, a partir del cual se dirigió un estudio descriptivo e interpretativo de los discursos, creencias y significaciones de docentes y estudiantes en torno a la enseñanza-aprendizaje del inglés en el Centro de Bachillerato Tecnológico Agropecuario No. 35 (CBTA No. 35) ubicado en el municipio de Texcoco, Estado de México. Los hallazgos de la investigación -obtenidos a partir de cuestionarios, entrevistas y un taller participativo- permitieron concluir que en las creencias, discursos y significaciones inconscientes de los educandos el inglés no es significativo y se refleja como un elemento ideológico alejado de su propia cosmovisión. Respecto a las intencionalidades y resistencias docentes y estudiantiles se determinó que constituyen áreas de oportunidad para que los educadores extiendan sus campos de acción y encuentren nuevos horizontes de formación orientados a que los estudiantes doten de sentido al aprendizaje del inglés como lengua extranjera.
\end{abstract}

Palabras clave: aprendizaje de idiomas, estrategias educativas, discurso, creencias, resistencia.

\section{Abstract}

The purpose of this article is to show the importance of the intentionalities and resistances that are built in the school environment as references to design effective English learning teaching strategies. The postulates are based on the integration of a theoretical model to understand the reality, which directed a descriptive-interpretative study of the teachers and students' discourses, beliefs and meanings regarding to English teachinglearning in the Centro de Bachillerato Tecnológico Agropecuario No. 35 (CBTA No. 35) located in the municipality of Texcoco, State of Mexico. The findings -obtained through questionnaires, interviews, and a participatory workshop- allow us to conclude that, in the unconscious beliefs, discourses and meanings of the students, English is not significant and is reflected as an ideological element, which is far removed from their own worldview. Regarding the intentionalities and resistances of teachers and students, it was determined that they constitute areas of opportunity for educators to expand their fields of action and find new horizons oriented to allow students to give meaning to the English learning as a foreign language.

Keywords: language learning, educational strategies, discourse, beliefs, resistance. 


\section{INTRODUCCIÓN}

El aprendizaje del inglés como lengua extranjera, por sus implicaciones en el desarrollo personal, social y académico de los estudiantes, ocupa un lugar destacado en la agenda educativa en México. Pese a ello, las evaluaciones de dominio del inglés muestran un claro rezago de los mexicanos en el desarrollo de las competencias comunicativas en dicha lengua extranjera. Al respecto, cabe destacar que el English Proficiency Index 2019 de Education First sitúa a México en el lugar 67 de 100 países evaluados, con un puntaje de 48.99 en una escala de 100 (Education First, 2019), de ahí que el gobierno haya estipulado la necesidad de concretar una política nacional con líneas de acción diversas dirigidas a contrarrestar los bajos niveles de aprendizaje del inglés.

Entre las propuestas de acción destacan: a) impulsar programas de capacitación y desarrollo profesional de los docentes de inglés (SEP, 2020); b) implementar un modelo de enseñanza mixta a través del cual los estudiantes tengan la oportunidad de tomar clases presenciales en su institución escolar y que estas sean complementadas con clases en línea a través de videoconferencias con maestros de inglés remotos (O’Donoghue, 2019), y c) optimizar los enfoques teóricos y aplicados sobre la enseñanza e investigar acerca de los procesos relacionados con el aprendizaje efectivo del inglés (García, Pérez, Martínez y Alfonso, 1998).

En lo que corresponde a la primera propuesta, la Secretaría de Educación Pública (SEP) diseñó el Programa Nacional de Inglés (2016) y la Estrategia Nacional de Inglés (2017), realizó la Jornada Virtual de Capacitación para Docentes y Asesores

Berenice Jaime Romero. Profesora-Investigadora en la Dirección General de Educación Tecnológica Agropecuaria y Ciencias del Mar (DGETAyCM) y en la Universidad del Pueblo, Plantel Tlacolula, México. Es doctorante en Ciencias en Educación Agrícola Superior en la Universidad Autónoma Chapingo, licenciada en Ciencias Políticas y Administración Pública, y maestra en Gobierno y Asuntos Públicos. Cuenta con especialidad en competencias docentes y especialidad en enseñanza y aprendizaje del inglés como lengua extranjera por la Universidad Pedagógica Nacional. Es miembro de la Asociación Mexicana de Maestros de Inglés (Mextesol) y de la Comisión Nacional de Maestros de Inglés de la DGETAyCM. Correo electrónico: bere_jaime@ hotmail.com. ID: https://orcid.org/0000-0001-8376-7474.

Willelmira Castillejos López. Profesora investigadora de la Universidad Autónoma Chapingo, México. Cuenta con estudios de licenciatura en lenguas inglesa y francesa por la Universidad Veracruzana, maestría en Lingüística por la Universidad Católica de Lovaina, Bélgica, y doctorado en Lingüística por la Universidad Autónoma Metropolitana. Sus áreas de interés se centran en las actitudes de hablantes monolingües y bilingües hacia variedades propias y ajenas, particularmente en escenarios de contacto lingüístico, asimismo ha trabajado en el ámbito educativo relacionado con la enseñanza y aprendizaje de lenguas extranjeras, de lo que extrae también una importante experiencia docente y de investigación. Correo electrónico: williecastillejos@hotmail.com. ID: https:// orcid.org/0000-0003-0500-3561.

Álvaro Reyes Toxqui. Profesor-investigador de la Universidad Autónoma Chapingo y de la Universidad Autónoma del Estado de México, México. Es licenciado en Sociología Rural por la Universidad Autónoma Chapingo, maestro en Desarrollo Rural por el Colegio de Postgraduados y doctor en Ciencias Agrarias por la Universidad Autónoma Chapingo. Entre sus publicaciones recientes se encuentra el libro Itinerarios sociales del cuerpo. Biopolítica y resistencia social (2019). Es candidato al Sistema Nacional de Investigadores. Correo electrónico: picaporte66@yahoo.com.mx. ID: https://orcid.org/0000-0003-0853-8932. 
Externos Especializados de Inglés (2020) y ha recomendado que todos los docentes de inglés obtengan la Certificación Nacional de Nivel de Idioma (CENNI). Por otra parte, en materia de oferta educativa, se ha incrementado el número de licenciaturas, especialidades, diplomados, maestrías y doctorados orientados a la enseñanza y el aprendizaje del inglés como lengua extranjera (COMIE, 2020). Asimismo, a fin de impulsar la superación y actualización de los docentes de lenguas, se han gestado diversos eventos académicos y se ha estimulado la integración de asociaciones, comités, redes y cuerpos colegiados.

Respecto a la segunda propuesta, la Universidad Abierta y a Distancia de México (UnADM) de la SEP y el Instituto Latinoamericano de la Comunicación Educativa (ILCE) han dado impulso al programa SEPA Inglés Online, el cual incorpora el uso de las tecnologías de la información y la comunicación para que los docentes y estudiantes desarrollen las cuatro habilidades del idioma (UnADM, 2021). A la par, la SEP ha establecido acuerdos con el Departamento de Comercio Internacional del Reino Unido en México (DIT), con la finalidad de que Little Bridge, Mangahigh y Twig Education, empresas británicas, proporcionen servicios educativos en inglés, a manera de donación, dentro de sus plataformas digitales (Robertson, 2021).

En torno a la tercera propuesta, existe una interesante producción teórica y empírica respecto al aprendizaje del inglés en México, misma que ha sido divulgada en los "Estados de conocimiento" publicados por el Consejo Mexicano de Investigación Educativa (COMIE, 2020) desde hace dos décadas. Los enfoques para el aprendizaje de idiomas que han sido más referenciados se enmarcan en el campo de la pedagogía, la lingüística aplicada, los enfoques o teorías de la enseñanza del inglés como lengua extranjera, segunda lengua o adquisición de segundas lenguas, seguidos por los basados en la sociología, la psicología y la psicopedagogía (COMIE, 2020).

Las líneas de investigación se han concentrado principalmente en los campos de enseñanza, aprendizaje, formación de docentes, creencias, y currículo, tecnología educativa e investigaciones en el área de la enseñanza de las lenguas extranjeras (Ávila, Carrasco, Gómez, Guerra, López y Ramírez, 2013). En dichos abordajes resulta paradójico que solo el 4\% de las investigaciones sobre los procesos de enseñanza y aprendizaje de las lenguas extranjeras en México se hayan realizado en torno al nivel medio superior (Ávila et al., 2013); sobre todo si se toman en cuenta las cifras de abandono escolar, los índices de eficiencia terminal y los bajos niveles de desempeño obtenidos por los bachilleres en las evaluaciones nacionales (Secretaría de Gobernación, 2020). En efecto, aunque existen diversos estudios en torno al diseño, implementación y evaluación de estrategias en materia de enseñanza del inglés, es evidente que existen dimensiones analíticas inherentes al aprendizaje de dicha lengua que se han dejado de lado. 
El propósito de este artículo es explorar los discursos, creencias y significaciones que tienen los docentes, y sobre todo los educandos, respecto al aprendizaje del inglés, considerando estos como referentes para diseñar estrategias didácticas efectivas para el aprendizaje del inglés como lengua extranjera. En consecuencia, se puntualiza la trascendencia de identificar: 1) las intencionalidades, definidas como las pretensiones o voluntariedades de los sujetos en torno a los objetos o sujetos de aprendizaje, y 2) las resistencias, entendidas como expresiones de contrapoder que manifiestan la necesidad de trastocar las lógicas educativas existentes en el proceso de enseñanza y aprendizaje del inglés.

\section{MODELO DE COMPRENSIÓN Y PROPUESTA CONCEPTUAL}

"El conocimiento de la realidad cada vez se vuelve más y más complejo, esotérico, paradójico, fragmentario, y abigarrado; la realidad se nos escabulle continuamente entre los dedos como si fuese agua, enredándonos en el cuento de no acabar" (Fregoso, 1996, p. 232). En este tenor, el propósito de visibilizar realidades intangibles llevó a la necesidad de analizar los discursos, creencias y significaciones insertos en los procesos de estudio a partir de un modelo de comprensión que permitiera reflexionar en torno a las categorías analíticas relevantes para este trabajo.

El modelo de comprensión de la realidad que se propuso (ver figura 1) está sustentado en las aportaciones de Foucault (1992), Searle (1992), Giroux (1992), Kemmis (1998), Zizek (1999), Van Dijk (1998), Lowe (1986), Ricouer (1994), Berger (1969), Geertz (2000), Vygotsky (1981) y Freire (1992). En consecuencia, se concibió como un marco de referencia que, a través de ciertas premisas, permitió leer las intencionalidades y resistencias presentes en los procesos de enseñanza y aprendizaje.

La primera premisa del modelo, basada en los estudios de Foucault (1992), es que las instituciones escolares son constructos en los que se hace el ejercicio de la dominación y se producen relaciones de poder en las que se encuentran inmersos directivos, docentes y estudiantes. Precisamente es en los procesos de enseñanza y aprendizaje donde se encuentran implícitas dichas relaciones, puesto que es en estos donde se sustenta el poder al establecer valores, estrategias y tácticas de acción. El docente lleva consigo intencionalidades que van más allá de la instrucción e incluso, como líder del proceso educativo, tiene la facultad de establecer castigos (tareas) y usar la represión (obligación) para controlar los saberes y las creencias sociales de los educandos. Los estudiantes, concebidos como centro del proceso educativo, también poseen intencionalidades y pueden constituirse como sujetos de poder capaces de ejercer su libertad y, por tanto, extrapolar resistencias a los mecanismos y dispositivos impuestos por el docente, los directivos e incluso la sociedad. 


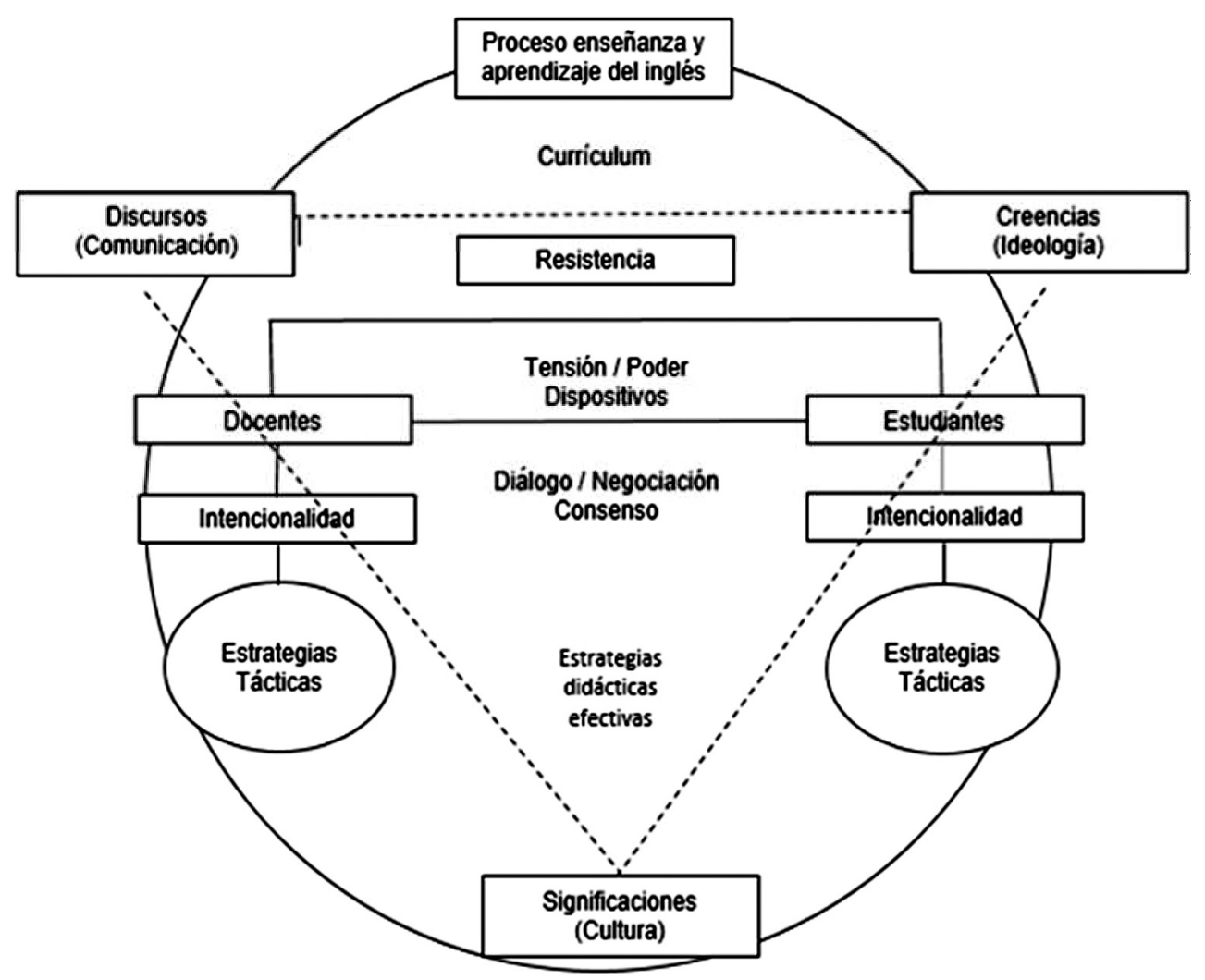

Figura 1. Modelo de comprensión de la realidad propuesto.

Fuente: Construcción personal.

La segunda premisa, parafraseando a Cuchumbé (2014), expone que los hechos institucionales educativos se asientan siempre en reglas constitutivas, y si se pretende comprender dichos hechos resulta esencial comprender la intencionalidad, pues esta refiere al modo de representación conceptual, en virtud del cual la representación es acerca de algo, está dirigida a algo. En este tenor, hay que entender a las intencionalidades, en términos de Searle (1992), como aquellas características de muchos estados y procesos mentales por las cuales estos se dirigen o son acerca de cosas del mundo. Asimismo es importante decir que las intencionalidades son el rasgo constitutivo del lenguaje, puente entre la mente y las cosas y el contexto (Arango, 2017).

La tercera premisa es que las resistencias educativas se pueden comprender, desde la analítica de Foucault, como las dimensiones subjetivas que reaccionan frente al poder y que interpelan una racionalidad dominante y la praxis que las acompaña (Castro, 2017). Al respecto, hay que hacer notar que las resistencias son productivas, en tanto generan nuevas formas de subjetividad que responden a los dispositivos de poder (estrategias y tácticas) obligándolos a una reactivación constante. Asimismo son reaccionarias y se modifican a partir de las limitaciones que les ofrece su adversario (Castro, 2017). 
La cuarta premisa es que para develar las intencionalidades y resistencias inherentes en los procesos educativos hay que redefinir, como diría Giroux (1992), la importancia del poder, la ideología, la cultura y la comunicación como construcciones centrales para comprender, a través de las creencias, los discursos y las significaciones, el rol de las escuelas, de los docentes y de los estudiantes dentro de la interacción compleja de la reproducción social. Ello conlleva vincular el comportamiento de los sujetos educativos con sus condiciones de relación históricas y acomete a un análisis crítico que involucre sensibilidad respecto a los intereses que dan lugar al desarrollo de una conciencia radical y a la acción colectiva crítica y emancipadora.

En cuanto al papel de la ideología, hay que hacer notar que "trasciende el nivel supuestamente no ideológico de la vida cotidiana y esta materialización de la ideología en un objeto concreto exterior pone en evidencia los antagonismos inherentes que no pueden ser reconocidos por la formulación ideológica explícita" (Zizek, 1999, p. 14). Consecuentemente, exteriorizar la ideología (ideas interiorizadas, sentimientos y experiencias) de los estudiantes y de los docentes para entender su funcionamiento en las escuelas conlleva el análisis de sus connotaciones, creencias (Van Dijk, 1998), percepciones (Lowe, 1986), relaciones sociales y formas de organización (Kemmis, 1998).

Respecto a la cultura, hay que enfatizar que es trascendente para la reflexión que se propone en este estudio dado que inmiscuye las visiones, estereotipos, actitudes, comportamientos y características compartidas del personal docente y del estudiantado. Esta es entendida, en términos de Geertz (2000), como la urdimbre de tramas de significación socialmente establecidas que nos permiten tener acceso al mundo conceptual en que está inserto el hombre y en virtud de las cuales la gente hace cosas tales como señales de conspiración y se adhiere a estas, o percibe insultos y contesta a ellos. Cierto, la cultura -sus imágenes y símbolos- es un elemento inherente a las personas, a sus pensamientos, idiomas, comportamientos, procesos de aprendizaje y desarrollo de competencias comunicativas (Vygotsky, 1981).

En las escuelas, y particularmente en los procesos de enseñanza y aprendizaje, se construyen redes comunicativas que permiten a los estudiantes repensar los sistemas establecidos a fin de reivindicar su papel como constructores de mundos de significado desde su propia circunstancia (Berger, 1969). La comunicación, expresada a través de discursos, códigos y gestos, es el medio a través del cual podemos visibilizar las creencias, las ideas, las significaciones y las percepciones de los sujetos del fenómeno educativo. De ello se infiere que la relevancia de la comunicación no radica solo en la comprensión sino primordialmente en las posibilidades de proposición que hacen factibles propuestas de mejora en torno al aprendizaje de cualquier campo de reflexión. 
La quinta premisa es que la adquisición lingüística del inglés como lengua extranjera depende no solo de la interrelación con el conocimiento y su vinculación con aspectos biológicos, sino que fundamentalmente es producto de las interacciones sociales que permiten su interiorización (Vygotsky, 1981). Esta se alcanza si el educando percibe el inglés no solo como un conjunto de signos orales y gráficos que se refieren al horizonte de lo otro sino como una herramienta útil y necesaria que pertenece al horizonte de lo deseable. Así que enseñar una lengua extranjera acomete a un proceso de estimulación lingüística que lleva a los estudiantes a relacionar signos-significados para después comunicar emociones, deseos e ideas en un ambiente lingüístico sociocultural.

La sexta premisa refiere que hay que entender las situaciones estratégicas como campos de batalla en los que el poder se desliza de acuerdo con una lógica que no es prioritariamente represiva, sino de carácter productivo (Castro, 2017). En consecuencia, el diseño de estrategias didácticas para el aprendizaje del inglés ha de partir del conocimiento de los elementos presentes en el proceso educativo, sus relaciones de poder, sus gestos de intencionalidad y resistencia y, sobre todo, ha de ser producto del consenso, la negociación y el diálogo entre los docentes y los educandos, a fin de estimular la autonomía docente y estudiantil y reivindicar, como expresa Freire (1992), el papel de la escuela como vehículo para alentar la emancipación social y la conquista de derechos.

La séptima premisa indica que es necesario conceptualizar, detectar el origen y concretar las formas de expresión que permiten aproximarse a las creencias (conscientes e inconscientes), discursos (actos del habla) y significaciones (sentido) de estudiantes y docentes en torno a la enseñanza y aprendizaje del inglés, a fin de visibilizar sus intencionalidades y resistencias (ver tabla 1). Las creencias son entendidas como conocimientos subjetivos generados por cada persona; los cuales no se fundamentan sobre la racionalidad sino más bien sobre los sentimientos, las experiencias y la ausencia de conocimientos específicos del tema con el que se relacionan (Moreno y Azcárate, 2003).

Los discursos acometen a la interacción social (Van Dijk, 1998), son entramados lingüísticos y no-lingüísticos, integrados por registros semióticos heterogéneos, que permiten expresar ideas, informaciones y estados afectivos sobre la enseñanza y el aprendizaje del inglés (Martínez-Otero, 2008). Las significaciones se definen como lo que representa en la práctica social una cosa o un fenómeno, de acuerdo con su esencia objetiva. Estas están determinadas por factores objetivos de la realidad (hechos, conceptos, conocimientos y patrones establecidos) y por factores subjetivos (deseos, tendencias, objetivos e intenciones sociales y personales del hombre) (Diccionario filosófico, 2020). 
Tabla 1. Categorías de aproximación al objeto de estudio.

\begin{tabular}{|c|c|c|c|c|}
\hline Objeto de estudio & Categorías teóricas & Categorías básicas & Origen & Formas de expresión \\
\hline \multirow[t]{3}{*}{$\begin{array}{c}\text { Intencionalidades } \\
\text { y resistencias }\end{array}$} & Ideología & $\begin{array}{l}\text { Creencias } \\
\text { (conscientes } \\
\text { e inconscientes) }\end{array}$ & $\begin{array}{l}\text { Conocimientos } \\
\text { subjetivos } \\
\text { de carácter social }\end{array}$ & $\begin{array}{l}\text { - Ideas interiorizadas } \\
\text { - Sentimientos } \\
\text { - Experiencias } \\
\text { - Percepciones }\end{array}$ \\
\hline & Comunicación & $\begin{array}{c}\text { Discursos } \\
\text { (actos de habla) }\end{array}$ & Interacciones sociales & $\begin{array}{l}\text { - Ideas } \\
\text { - Manifestaciones discursivas } \\
\text { - Expresiones del lenguaje }\end{array}$ \\
\hline & Cultura & $\begin{array}{l}\text { Significaciones } \\
\text { (sentido) }\end{array}$ & $\begin{array}{c}\text { Prácticas sociales } \\
\text { y culturales }\end{array}$ & $\begin{array}{l}\text { - Deseos } \\
\text { - Intenciones } \\
\text { - Actitudes }\end{array}$ \\
\hline
\end{tabular}

Fuente: Elaboración propia.

\section{ENFOQUE METOdOLÓGICO}

La investigación partió de la premisa de Cook y Reichardt (1986) de que el empleo complementario de métodos cualitativos y cuantitativos contribuye a corregir los inevitables sesgos presentes en cualquier método. Siguiendo dicho postulado, el enfoque investigativo fue de naturaleza mixta con un diseño explicativo secuencial (DExPLIS), por lo que se llevaron a cabo estrategias cuantitativas y cualitativas para la recolección y análisis de datos a fin de obtener mayor amplitud, profundidad, diversidad y riqueza interpretativa respecto al tema de interés.

Se decidió trabajar con la comunidad escolar del CBTA No. 35 ubicado en Texcoco, Estado de México, dado que en dicha institución la asignatura de Inglés presentaba los más altos índices de reprobación escolar (39\%) de la región (SEMSDGETA, 2019). Los participantes fueron estudiantes (27 hombres y 25 mujeres) y docentes (cuatro hombres y cuatro mujeres) de primer semestre de la carrera de Técnico Agropecuario. En cuanto al perfil de los estudiantes, la edad promedio fue de 15 años; 98.07\% eran solteros; 82.7\% indicó ser de estrato económico bajo-alto (ingreso familiar entre $\$ 4,500$ y $\$ 9,000$ ), $11.5 \%$ de estrato bajo-bajo (ingreso familiar menor a $\$ 4,000$ ) y $5.8 \%$ medio-bajo (ingreso familiar entre $\$ 9,000$ y $\$ 18,000$ ); además el 59.6\% manifestó provenir de familias de floricultores y 34.6\% tener actividades, sin remuneración económica, de apoyo en la siembra y venta de flores. Por su parte, la edad promedio de los docentes era de 43 años; todos indicaron ser de estrato económico medio-bajo; el 50\% con plaza de 20 horas, el 25\% con plaza de 30 horas y el otro $25 \%$ con plaza de tiempo completo.

El estudio se dividió en tres fases. En un primer momento se realizó una investigación documental y teórica enfocada en encontrar un modelo de comprensión de la realidad permisible para dirigir la disertación. La segunda etapa constituyó el 
trabajo de campo, el cual implicó la recolección de datos a través de la aplicación de un cuestionario escrito a estudiantes, un cuestionario digital a docentes, un taller participativo con estudiantes y dos entrevistas a profundidad con docentes. Finalmente se realizó el análisis e interpretación de los hallazgos a partir de un tratamiento estadístico y una examinación multinivel secuencial para relacionar categorías, todo ello con el cometido de enunciar las conclusiones del estudio.

El cuestionario a estudiantes constó de 25 preguntas cerradas y dos abiertas enfocadas en conocer sus experiencias y percepciones (creencias y significaciones) en torno al aprendizaje del inglés. De acuerdo con la prueba estadística de análisis no paramétrico Chi cuadrada $\left(\mathrm{X}^{2}\right)$, dicho instrumento presentó una confiabilidad aceptable al tener un valor 0.720 y buena por su valor de 0.823 basada en elementos estandarizados. El cuestionario se aplicó a 52 integrantes de los dos grupos de primer semestre adscritos al CBTA No. 35. La elección de los informantes se realizó a través del método de muestreo por conveniencia.

El cuestionario digital para docentes se realizó a través de la herramienta formularios de Google y estuvo integrado por 45 cuestionamientos. El método propuesto para esta aplicación fue el de censo, la liga para contestar dicho instrumento se remitió vía email a los ocho docentes de la asignatura de Inglés del CBTA No. 35, recibiendo la contestación del 75\% de los implicados. Las preguntas manifiestas en este estaban orientadas a identificar los significados que los docentes dan a la enseñanza y el aprendizaje del inglés, las problemáticas a las que se enfrentan y su disposición a la reinvención.

El taller participativo se realizó mediante una guía de discusión y tuvo una duración de hora y media. En este participaron los 52 estudiantes de los dos grupos seleccionados previamente a través del muestreo por conveniencia. Las preguntas detonadoras fueron: 1) ¿Cómo han sido sus experiencias en torno al aprendizaje del inglés?, 2) ¿Qué creen que ha fortalecido o dificultado su aprendizaje del inglés?, 3) ¿Qué significa para ustedes aprender inglés? Su cometido fue socializar y reflexionar colectivamente los discursos, creencias y significaciones de los estudiantes en torno a la enseñanza-aprendizaje del inglés para determinar sus resistencias e intencionalidades. El taller fue grabado y posteriormente se realizó su relatoría para proceder a su análisis.

Las entrevistas a profundidad con los dos docentes se dirigieron a través de una guía de entrevista, cuyas preguntas fundamentales fueron: 1) ¿Qué significa para usted enseñar inglés? y 2) ¿Cómo se siente (nivel de satisfacción) en el cumplimiento de su rol como docente? La selección de los docentes entrevistados se realizó a través del método de muestreo por conveniencia teniendo como parámetro que los informantes fueran docentes de la asignatura de Inglés I en la Extensión Texcoco. Las entrevistas fueron grabadas y posteriormente transcritas. La trascendencia de dichas entrevistas 
radicó en que brindaron información importante para determinar las intencionalidades y resistencias de los docentes en torno a la enseñanza y el aprendizaje del inglés.

Para la concentración y análisis de los datos cuantitativos se diseñó una base de datos, se determinaron medidas de frecuencias, tablas cruzadas y pruebas estadísticas como Chi cuadrada. El procesamiento de la información fue realizado con la aplicación de hojas de datos Excel y el paquete estadístico SPSS versión 25. Por otro lado, los datos cualitativos fueron analizados temáticamente por medio de la estrategia de codificación abierta, el establecimiento de códigos conceptuales y la codificación axial (construcción de redes de relaciones), todo ello haciendo uso del programa Atlas.ti versión 8.

\section{RESUlTADOS Y DISCUSIÓN}

Los resultados vertidos son producto de un análisis global cuyo propósito es considerar las siete premisas expuestas en el modelo de comprensión de la realidad propuesto, a fin de brindar una visión general de las creencias, discursos y significaciones de los estudiantes en torno a la enseñanza y el aprendizaje del inglés. A través de un proceso descriptivo se destacan los aspectos más relevantes asumidos por los estudiantes y los docentes en torno a las categorías de aproximación al objeto de estudio.

\section{Intencionalidades y resistencias de los estudiantes en torno a la enseñanza y el aprendizaje de inglés}

Las creencias de los estudiantes en torno a la enseñanza y el aprendizaje del inglés permitieron corroborar la séptima premisa, puesto que en efecto están basadas en sus experiencias, las cuales de acuerdo con el $63.3 \%$ de los mismos han sido negativas. $\mathrm{Al}$ respecto los estudiantes expresaron que las experiencias negativas tenían relación con las malas calificaciones obtenidas en sus evaluaciones $(84.6 \%)$, las interacciones con sus compañeros en clase $(69.2 \%)$ y la reprobación (48.1\%). El 30.8\% relacionó sus experiencias negativas con los docentes y el $25 \%$ con sus escuelas anteriores. De hecho se encontró a través de una tabla cruzada que el 71.4\% de los estudiantes que habían tenido experiencias negativas consideraban muy difícil o difícil aprender inglés.

Las experiencias negativas de los estudiantes en torno al aprendizaje del inglés, expresadas a lo largo del taller participativo, permitieron asociar códigos y buscar códigos de patrones, a través de lo cual se perfiló que dichas experiencias estaban relacionadas con los siguientes códigos: 1) aburrimiento, 2) no entendían las explicaciones de los docentes, 3 ) las actividades eran las mismas (copiar textos en la libreta y traducirlos con el diccionario o contestar ejercicios en hojas o en la libreta), 4) no tenían maestros, 5) cambiaban mucho de maestro, 6) la actitud de los maestros no era favorable y 7) sus compañeros de clase influían en esas malas experiencias, al interrumpir constantemente las clases, ser apáticos e irresponsables con las tareas 
escolares. Algunas citas que fueron extraídas del taller participativo y que se eligieron, dado que eran más descriptivas y permitían evidenciar el sentir del estudiantado, permitieron corroborar la quinta premisa en cuanto a las experiencias de los educandos en el aprendizaje del inglés. Estas son:

[Participante 1] En la primaria aprendí algo de inglés, allí fue donde conocí a mi maestro favorito, el maestro L.., él me dio el medio año de tercer grado, ese maestro era psicólogo y unos días nos daba musicoterapias. Pasé a cuarto grado y me enseñaron algunas palabras en inglés donde hasta ahora las pronuncio bien; en quinto grado cambié de maestro como tres veces porque ninguno aguantó el desastre de mis compañeros. En sexto grado me tocó el bendito maestro A..., un maestro que me odiaba... gracias a él obtuve un promedio bajo, pero no me importó y me reía de él en su cara... En segundo grado de secundaria tuve problemas con un maestro porque no le entendí a su clase y mandó llamar a mi mamá; mi papá fue, pero no quiso hablar con él porque le tenía miedo. En tercero de secundaria levanté bien chido mi calificación de inglés y entré a un concurso de deletreo, ese día fue mi día. Quedé en uno de los cinco finalistas y gané.

[Participante 2] En tercero de primaria fue cuando conocí la materia de Inglés con la maestra X, ella también me dio en cuarto grado. En quinto grado no me daban inglés si no pagaba mensualmente la clase. En sexto de primaria no quise que mis padres pagaran el inglés porque no me enseñaban nada. En primero de secundaria las clases de inglés eran muy aburridas porque la maestra se la pasaba tomando selfies con los alumnos. En segundo la maestra era estricta, nos ponía a entablar conversaciones y aprendí a pronunciar algunas palabras. En tercero la maestra era la misma que la de segundo y nos dejó hacer la obra de La bella durmiente frente a toda la escuela, lo cual fue relevante.

[Participante 3] Mi experiencia en el inglés ha sido difícil porque no le entiendo muy bien.

Resultó de interés conocer las percepciones de los estudiantes en torno a su nivel de dominio de cada habilidad lingüística para ir acercándose a sus discursos. El 59.6\% de los estudiantes refirió que su dominio de comprensión auditiva era regular, el 25\% malo y el 15.4\% bueno. En cuanto a lectura el 55.8\% determinó que era regular, $23.1 \%$ bueno, $19.2 \%$ malo y el 1.9\% muy malo. Respecto a expresión oral el 53.8\% expresó que su dominio era regular, $26.9 \%$ malo, $11.5 \%$ bueno, $3.8 \%$ muy malo y $3.8 \%$ muy bueno. En torno a la escritura el 48.1\% determinó que su nivel era regular, 30.8\% malo, $19.2 \%$ bueno y $1.9 \%$ muy bueno. A través de la prueba estadística de análisis no paramétrico Tau_b de Kendall y la prueba Rho de Spearman se rechazó la hipótesis nula de que las percepciones emitidas en cada habilidad eran independientes pues no tenían relación, y se aceptó la hipótesis alternativa que expresaba que existía algún grado de relación (positiva media y débil) entre las mismas.

El 51.9\% de los educandos expresó que consideraba muy difícil aprender inglés, el $32.7 \%$ regular, el 13.5\% muy difícil y solo el 1.9\% fácil. La habilidad del idioma que consideraban de mayor dificultad era escritura (32.6\%), seguida de lectura (28.8\%), expresión oral (28.8\%) y comprensión auditiva (21.1\%). Entre los aspectos que refirieron dificultan o les dificultaron el aprendizaje de dicha lengua se encuentran: estra- 
tegias didácticas (38.5\%), actividades formativas de apoyo (tareas, quizzes, actividades en línea, etc.) (19.2\%), material didáctico (manuales de trabajo, audios, video, etc.) (3.8\%), número de estudiantes en el salón (3.8\%), tiempo disponible para estudiar la materia (13.5\%), horario en que se imparte la asignatura (11.55), ubicación del salón de clases $(1.5 \%)$, no-utilización de herramientas tecnológicas (software interactivos, audios, videos, traductores electrónicos y películas) (1.9\%), no les gusta pues no le entienden $(5.8 \%)$.

Los aspectos relacionados con las dificultades del inglés se corroboraron en el taller participativo a través de la técnica presencia-ausencia, de esta forma se encontró que para el $70 \%$ de los estudiantes el inglés representaba un problema o dificultad. Entre las causas de dicho hecho se evidenció la relevancia de la sexta premisa, pues hicieron presencia las categorías forma de enseñar, falta de dinámicas, mismas actividades, clases aburridas, el maestro no dominaba los temas, mismas que se categorizaron como parte de la categoría predeterminada estrategias didácticas de enseñanza. Asimismo los estudiantes refirieron problemas para pronunciar, no conocer el vocabulario, dificultades para entender las estructuras de las oraciones, lo cual se ubicó dentro de la categoría estrategias didácticas de aprendizaje. En este rubro también se mencionaron categorías como falta de asesorías sobre el tema y no tener internet (para ver videos de tarea o practicar en plataformas como Duolingo o YouTube).

Cuando se les cuestionó sobre la utilidad de aprender inglés para su vida el 34.6\% de los estudiantes respondió que era muy útil, el 51.9\% que era útil, el 7.7\% regular y el 5.8\% no muy útil. En consecuencia, en términos discursivos, para el 86.5\% de los educandos el inglés es útil para la vida. Los resultados anteriores se ratificaron a lo largo del taller participativo, pues los estudiantes expresaron que aprender inglés les permitiría comunicarse y relacionarse con personas de otros países, poder entender canciones, películas o videos en inglés, o les daría estatus en su vida social y viajar al extranjero.

En cuanto a la utilidad de aprender inglés para su carrera profesional, el 63.5\% de los estudiantes estipuló que era muy útil, el 26.9\% que era útil, el 7.7\% regular y el 1.9\% no muy útil. Como se puede ver, las percepciones se concentraron en que el aprendizaje es útil para la vida profesional (90.4\%), lo cual también se ratificó con las aseveraciones vertidas en el taller participativo en cuanto a que aprender inglés les daría la oportunidad de obtener becas para estudiar en el extranjero, graduarse de una licenciatura, acceder a un posgrado, obtener mejores empleos y tener un mejor salario. Se encontró que existe una correlación positiva media entre las percepciones de la utilidad del inglés para la vida y la utilidad del inglés para la carrera profesional gracias a la prueba Tau_b de Kendall, la cual tuvo un valor de 0.526.

Las tres principales razones (intencionalidades) que expresaron los estudiantes tener para aprender inglés fueron: para su futuro ejercicio profesional (40.4\%), para 
viajar a diferentes países $(23.1 \%$ ) y para tener una mejor comprensión del estilo de vida de los países en que es hablado (13.5\%). Las razones menos importantes fueron para conocer una mayor variedad de personas en su vida (1.9\%), para conseguir una beca para estudiar en el extranjero $(1.9 \%)$ y porque les gustaría vivir en un país donde se habla inglés (3.8\%).

Resultó contradictorio a lo antes mencionado que en la interrogante sobre qué significado tenía aprender inglés el 40.4\% de los estudiantes no contestó, el 1.9\% no sabía que significaba, para el 1.9\% el inglés no significaba nada y para el $7.7 \%$ el inglés significaba dificultad. Ahora bien, para el 17.3\% el inglés significaba algo importante, para el 15.4\% algo útil para la vida académica, profesional o social, para el 5.8\% estudiar y repasar, para el 3.8\% aprender y usar el idioma, y para el 5.8\% una necesidad.

En el taller participativo los estudiantes ratificaron que consideraban que aprender inglés significaba prestigio social, mejores oportunidades académicas o profesionales. No obstante, expresaron que no se sentían marginados ni social ni lingüísticamente desfavorecidos por el hecho de no aprender inglés, puesto que ni sus parientes cercanos ni sus amigos o vecinos usan frecuentemente dicha lengua para comunicarse en la vida común, lo cual perfiló la trascendencia de la cuarta premisa. El 90\% de los participantes dijo que el inglés no estaba presente en sus comunicaciones cotidianas, era solo parte de la escuela y, aunque a algunos les gustaba escuchar canciones o ver películas en inglés, no le daban mucha importancia a entender lo que estas enunciaban.

Como resultado de los hallazgos se llegó a la conclusión de que el inglés se vislumbra como algo importante en el discurso consciente de los estudiantes, lo cual se justifica en los discursos que les han vendido los medios de comunicación, los programas educativos y las escuelas en el marco de la globalización y el neoliberalismo. Sin embargo, la realidad es que en las creencias, discursos y significaciones inconscientes de los educandos el inglés no es significativo y se refleja como un elemento ideológico alejado de su propia cosmovisión, lo cual afirma la segunda premisa. El inglés es percibido como "la lengua extranjera", "la lengua de los turistas, los gringos y los güeritos"; de ninguna forma significa el lenguaje común que les permite sobresalir, romper barreras comunicativas o acceder al mercado laboral en su futuro inmediato.

Tomando en cuenta lo anterior, se observó que los estudiantes presentan resistencias al aprendizaje del idioma, a las estrategias, los mecanismos y dispositivos utilizados en la enseñanza de lenguas, todo ello producto de sus experiencias y dificultades en los procesos educativos, lo cual alude a la tercera premisa. En consecuencia, las intencionalidades de la mayoría de los estudiantes en cuanto al aprendizaje del inglés se reducen a acreditar las asignaturas, pues el inglés no ocupa un lugar destacado en su agenda diaria, semanal o mensual.

Para finalizar este apartado, hay que hacer notar que las aseveraciones y conclusiones vertidas no deben dejar de lado el hecho de que los rasgos personales de cada 
estudiante influyen en su modo de pensar y de sentir y, por tanto, pueden llevarlos a poseer intencionalidades y resistencias divergentes a los del resto de la comunidad escolar (como es el caso de los dos estudiantes de alto desempeño que participaron en el taller), lo que provoca singularidades que, aunque no son objeto de este estudio, son interesantes de analizar tal y como lo afirma la primera premisa.

\section{Intencionalidades y resistencias de los docentes en torno a la enseñanza y el aprendizaje del inglés}

El cometido de develar las intencionalidades y resistencias de los docentes llevó a cuestionarlos sobre lo que significa para ellos enseñar inglés y cómo se sentían (nivel de satisfacción) en el cumplimiento de dicho rol. Así mismo se les interrogó sobre los problemas que enfrentan como docentes para desarrollar las habilidades lingüísticas de inglés, si consideran las características, los estilos de aprendizaje, los intereses y necesidades de los estudiantes para el diseño de sus estrategias didácticas y qué estrategias utilizan. Por último se les preguntó su disposición para diseñar e implementar nuevas estrategias en el proceso de enseñanza-aprendizaje.

En cuanto a lo que significa enseñar inglés, el 50.1\% expresó que significa proporcionar conocimientos, valores y habilidades a los estudiantes para que puedan expresarse correctamente haciendo uso del inglés; el 33.4\% refirió que significa fomentar en los estudiantes el descubrimiento y aprendizaje de una lengua extranjera, y el otro $16.7 \%$ crear entornos de aprendizaje efectivos que lleven a los estudiantes a comunicarse en una lengua extranjera en situaciones cotidianas. Al respecto se seleccionaron las siguientes dos citas ya que permitieron corroborar los códigos antes mencionados.

[Docente 1] Como docente de una escuela tecnológica agropecuaria considero que el significado de la enseñanza del inglés va más allá de enseñar palabras o de traducir. A diferencia de otras materias, enseñar o aprender un idioma implica aprender una cultura, abrirles los ojos a los muchachos de que hay algo más allá de donde conocen. Enseñar inglés implica hablar de canciones, películas y, por tanto, sumergirse en aspectos culturales. Enseñar implica ayudar a nuestros estudiantes a pensar diferente, abrir su horizonte de posibilidades y oportunidades para que vean las cosas de otra manera. El inglés como lengua franca le abre las puertas al mundo.

[Docente 2] Para mi enseñar inglés a nivel bachillerato tiene un significado muy importante porque me da la oportunidad de poder compartir a los estudiantes las cosas que yo sé, los conocimientos, en este caso enfocados a una lengua extranjera como lo es el inglés. Sobre todo, nuestra misión como maestros, desde mi perspectiva, pues tiene que ser concientizar a los estudiantes de que el inglés no es solo una asignatura sino que es la puerta de acceso hacia otra cultura, conocer otras formas de vida a través del idioma, otras costumbres. Para mí lo importante es la riqueza cultural que conlleva aprender un idioma.

Considerando lo anterior es posible expresar que las intencionalidades de los docentes se dirigen a que los estudiantes perciban el aprendizaje del inglés dentro 
del horizonte de lo deseable. Su propósito no es solo enseñar un idioma sino abrir vetas de reflexión y estructurar significados y usos del inglés dentro del entramado cultural de los angloparlantes. En efecto, para ellos enseñar es dotar de significado al aprendizaje del inglés para resignificar la realidad de los educandos de acuerdo con las condiciones y necesidades del sistema mundo.

Cuando se cuestionó a los docentes sobre su sentir (nivel de satisfacción) en el cumplimiento de su labor, el 83.3\% manifestó que disfrutaba ser docente y se sentía bien (muy satisfecho) con su propio desempeño. Pese a ello, el 50\% de los docentes expresó que se sentía mal (poco satisfecho) en cuanto a los aprendizajes de los estudiantes en el desarrollo de las habilidades lingüísticas; el 33.3\% se sentía regular (satisfecho) y el 16.7\% se sentía bien (muy satisfecho). Considerando ambos aspectos, resultó interesante llevar a cabo la prueba Tau_b de Kendall, obteniendo esta un valor de -.674, con lo que se determinó que existía asociación negativa media entre el sentir de los docentes en su propia labor como educadores (satisfacción respecto a la enseñanza) y su sentir con respecto a los aprendizajes de los estudiantes, por tanto se aceptó la hipótesis nula.

Otro aspecto que se consideró importante, tomando en cuenta los resultados previos, fue conocer los problemas que enfrentan los docentes con los estudiantes. El 33.3\% refirió que tenía hasta 40\% de reprobados por semestre, otro 33.3\% presentaba en promedio $30 \%$ de reprobación, $16.7 \%$ tenía $20 \%$ de reprobación y otro 16.7\% manifestó tener 15\% de reprobación. Los docentes detectaron que la habilidad lingüística del inglés que consideran de mayor dificultad para desarrollar en los estudiantes es comprensión auditiva (50\%), seguida de expresión oral (33.3\%) y lectura $(16.7 \%)$. Entre los principales problemas a los que se enfrentan día a día están irresponsabilidad en el cumplimiento de tareas y trabajos extraescolares (50\%), que los estudiantes no practiquen el idioma ni repasen ni estudien lo visto en clase $(33.3 \%)$ y la falta de interés en el idioma (16.7\%).

$\mathrm{Al}$ respecto los entrevistados enunciaron lo siguiente:

[Docente 1] El principal problema al que me enfrento es la reprobación y el bajo desempeño de los estudiantes. A pesar de que el examen de conocimientos solo tiene un valor de $40 \%$ los estudiantes se resisten a cumplir con los productos que se les solicita. Solo cuatro o cinco estudiantes participan continuamente, cuando se les deja hacer presentaciones orales no las ensayan y no viven la comunicación, es como si únicamente se les dejara leer, y ni siquiera con una pronunciación aceptable; además, únicamente los estudiantes destacados se preocupan por hacer sus tareas, el resto las copia, y mal. Si se les deja hacer pequeñas entrevistas en inglés con tres o cuatro interrogantes la mayoría no las hace. El esfuerzo de los estudiantes se reduce a las tres horas de clase y después de ese tiempo el inglés no ocupa ningún espacio dentro del plan de vida semanal de la mayoría. Por si fuera poco, las autoridades solicitan disminuir los índices de reprobación y ello lleva a que terminemos aprobando a los estudiantes que tienen muy bajos desempeños. 
[Docente 2] Para mí uno de los problemas de mayor envergadura es que a los estudiantes no les gusta el inglés, quizás por las experiencias didácticas que han tenido a lo largo del aprendizaje de este, por el hecho de que algunos tienen abuelos que hablan náhuatl y les gustaría mejor aprender dicha lengua o porque simplemente no les llama la atención. Lo cierto es que los estudiantes se resisten, para ellos es una lengua llena de contrastes. No entienden por qué nosotros decimos que tenemos tantos años y que en inglés se expresa que tantos años somos viejos; para ellos es algo que contradice no solo la estructura oracional sino sus propias referencias lingüísticas. Además los estudiantes no tienen estrategias para el aprendizaje, lo que antes llamábamos hábitos de estudio, y por si fuera poco, dentro del ámbito de sus interacciones sociales muy pocos tienen la posibilidad de practicar el idioma. Con todo ello, es difícil que los estudiantes tengan un aprendizaje óptimo y van pasando con los aprendizajes mínimos.

En lo que respecta a si los docentes toman en cuenta las características, los estilos de aprendizaje, los intereses y necesidades de los estudiantes para el diseño de sus estrategias didácticas el $66.7 \%$ de los docentes refirió que sí lo hacen. En este punto los entrevistados enunciaron que el hecho de que la institución les solicite hacer entrega de las estrategias didácticas antes de iniciar el curso escolar limita planear estrategias basadas en las características de los estudiantes de primer semestre, pero de alguna forma su experiencia de trabajo les permite tener un enfoque global de los intereses y necesidades de los estudiantes.

Entre las estrategias que utilizan en clase están: disciplina (80\%), solicitud de trabajos escritos $(33.3 \%)$, investigaciones, $(16.7 \%)$ juego de roles $(16.7 \%)$, construcción de mapas mentales $(16.7 \%)$, relatorías $(16.7 \%$ ) y aprendizaje basado en retos $(33.3 \%)$. Al respecto los docentes expresan que trabajan con actividades seleccionadas de libros, las cuales se enfocan en prácticas, sobre todo de gramática. También llevan al aula audios para la práctica de la comprensión auditiva, lecturas, y dejan ejercicios de escritura.

El 100\% de los docentes manifestó su disposición para diseñar e implementar nuevas estrategias en el proceso de enseñanza-aprendizaje a fin de contribuir al desarrollo de las habilidades lingüísticas del inglés en sus estudiantes. En este tenor, expresaron que para poder cumplir dicho cometido necesitaban capacitaciones no solo en estrategias didácticas sino también en estrategias de evaluación, habilidades socioemocionales, teorías del aprendizaje de lenguas extranjeras, estilos de aprendizaje, microenseñanza, recursos didácticos, manejo de las tecnologías de la información y la comunicación y manejo psicológico.

En las entrevistas los docentes refirieron que desafortunadamente no tenían mucho tiempo para su formación y los cursos propuestos por la nueva escuela mexicana no estaban dirigidos para los docentes de inglés. Por dicha razón, manifestaron que lograr los propósitos educativos en el área de inglés era un reto que requería su esfuerzo personal, pero también apoyo de las autoridades y padres de familia para detectar las verdaderas problemáticas en los procesos de enseñanza y aprendizaje. 
Todo lo anterior dejó entrever que en el discurso consciente las intencionalidades de los docentes son acordes a las determinaciones expuestas por el marco normativo institucional, así como con los planes y programas emitidos por la Subsecretaría de Educación Media Superior; no obstante, es un hecho que sus intencionalidades visibles van más allá, pues incluso tratan de generar, a través del aprendizaje del inglés, un cambio en las formas de pensar de los educandos. En el discurso inconsciente los docentes desean mantener su statu quo para cumplir con el control en el aula a través de dispositivos - como son las estrategias de evaluación- que les permitan hacer frente a las resistencias de los estudiantes para así cumplir con los índices de aprobación requeridos por la institución.

\section{Conclusiones}

Este artículo ha pretendido ser una provocación para interpelar las intencionalidades y resistencias en el proceso de enseñanza y aprendizaje del inglés en un contexto y espacio temporal específicos. Simultáneamente, ha postulado que dichas intencionalidades y resistencias son referentes para diseñar estrategias didácticas efectivas que ayuden a contrarrestar los bajos índices obtenidos por los mexicanos en cuanto al dominio del inglés.

La exploración de la realidad material se realizó a través de un proceso investigativo de naturaleza mixta en el que los sujetos de estudio fueron tanto estudiantes como docentes. Los hallazgos permitieron corroborar y sustentar algunas premisas teóricas que se postularon con la finalidad de conducir el análisis. Asimismo se logró identificar algunos elementos de interés que pueden ser objeto de estudios posteriores e incluso justificar propuestas de intervención educativa.

En primer lugar, se postuló que el proceso de la enseñanza y aprendizaje del inglés es en sí mismo un juego estratégico que implica reinventar la didáctica para lograr la emancipación de los educandos. Consecuentemente, la dinámica escolar no solo debe percibirse como una realidad sino como un sistema de sentido con códigos socialmente producidos e interiorizados que inmiscuye intencionalidades y resistencias, en el marco de las reglas constitutivas de las escuelas.

En segundo lugar, se argumentó que la ideología inherente a las creencias, la cultura percibida en las significaciones y la comunicación manifiesta en los discursos son elementos indispensables para develar las intencionalidades y resistencias en torno al aprendizaje del inglés. Esto se justifica en el hecho de que la adquisición de una lengua solo es posible si se da dentro del campo de las interacciones sociales en donde subyacen elementos culturales y lógicas operativas para la emisión y recepción de cualquier mensaje.

La conclusión a la que se llega a lo largo de esta investigación es que hay que cuestionarse profundamente sobre qué hacer para confrontar las implicaciones de la 
praxis educativa, las cuales resaltan la incapacidad de conciliar las intencionalidades de los docentes y el sistema educativo con las intencionalidades de los estudiantes adolescentes sumergidos en la inmediatez del ahora. Para ello, se propone que los docentes de inglés centren sus focos de atención en los elementos encubiertos no observables directamente (contenidos inconscientes, rasgos de los estudiantes y motivaciones) que dificultan su labor y, a partir de ello, diseñen estrategias didácticas no solo de memoria, cognitivas o de compensación, sino fundamentalmente metacognitivas, afectivas y sociales.

En definitiva, hay que reinventar la enseñanza y el aprendizaje del inglés y, para ello, hay que partir del hecho de que las intencionalidades y resistencias del estudiantado son producto del mundo simbólico que ha construido su cultura. En consecuencia, el diseño de estrategias didácticas, que guíen la construcción de entornos significativos de aprendizaje del inglés, hace indispensable concebir actividades y seleccionar recursos que estén en consonancia con el contexto, los conocimientos, los intereses, los estilos de aprendizaje, las emociones, las creencias, los valores, los discursos y las motivaciones de los educandos. Es imprescindible incluir dinámicas de concentración, activación y de trabajo en equipo. Asimismo hay que incursionar en el uso de metodologías propicias al qué, cómo y por qué de los procesos educativos que se gestan en torno al aprendizaje de una lengua extranjera.

\section{REFERENCIAS}

Arango, G. J. (2017). La teoría de la intencionalidad de John Searle. Sophia, Colección de Filosofía de la Educación, (22), 79-98. Recuperado de: https://www.redalyc.org/ pdf/4418/441849567003.pdf.

Ávila, A., Carrasco, A., Gómez, A. A., Guerra, M. T., López, G., y Ramírez, J. L. (2013). Una década de investigación educativa en conocimientos disciplinares en México (2002-2011): matemáticas, ciencias naturales, lenguaje y lenguas extranjeras. México: ANUIES-COMIE.

Berger, P. (1969). El dosel sagrado. Para una teoría sociológica de la religión. Buenos Aires: Amorrortu Editores.

Castro, R. (2017). Foucault y la resistencia. Una gramática del concepto. Contrastes. Revista Internacional de Filosofía, 22(1), 45-63.

COMIE (2020). Consejo Mexicano de Investigación Educativa A.C. Recuperado de: http://www.comie.org.mx/ v5/sitio/.

Cook, T. D., y Reichardt, C. S. (1986). Métodos cualitativosy cuantitativos en investigación evaluativa. Madrid: Ediciones Morata.
Cuchumbé, N. (2014). Lenguaje y rasgos constitutivos en John Searle: aporte al estudio de los procesos mentales. Praxis Filosófica, (38), 71-87. Recuperado de: http://praxisfiloso fica.univalle.edu.co/index.php/ filosofica/article/view/2842/2851.

Diccionario filosófico (2020). Significacion y sentido. Barcelona.

Education First (2019). EF English Proficiency Index. Recuperado de: https://www.ef.com/assetscdn/ WIBIwq6RdJvcD9bc8RMd/legacy/_/ / / media/ centralefcom/epi/downloads/full-reports/v7/efepi-2017-english.pdf.

Foucault, M. (1992). Vigilary castigar: nacimiento de la prisión. Madrid: Siglo XXI.

Fregoso, A. (1996). El sentido común: casa de espejos. Libro II. Texcoco: Universidad Autónoma Chapingo.

Freire, P. (1992). Pedagogía del oprimido. Madrid: Siglo XXI. García, R., Pérez, F., Martínez, T., y Alfonso, V. (1998). Estrategias de aprendizaje y enseñanza del inglés como segunda lengua en contextos formales. Revista 
de Educación, (316), 257-269. Recuperado de: http:// www.educacionyfp.gob.es/dam/jcr:003c01ff-15824409-9c82-247963ef9c5a/re3161400464-pdf.pdf.

Geertz, C. (2000). La interpretación de las culturas. Barcelona: Gedisa.

Giroux, H. (1992). Teoría y resistencia en educación. México: Siglo XXI Editores.

Kemmis, S. (1998). El curriculum: más allá de la teoría de la reproducción. España: Morata.

Lowe, D. M. (1986). Historia de la percepción burguesa. México: FCE.

Martínez Escárcega, R. (2009). Poder y resistencia en la cotidianidad de la vida escolar. X Congreso Nacional de Investigación Educativa | área 14: práctica educativa en espacios escolares. Veracruz, Veracruz, México: COMIE.

Martínez-Otero, V. (2008). Discurso educativo y formación docente. Revista Educação em Questão, 33(19), 9-34. Recuperado de: https://redalyc.org/articulo. oa?id $=563959965001$.

Moreno, M., y Azcárate, C. (2003). Concepciones y creencias de los profesores universitarios de matemáticas acerca de la enseñanza de las ecuaciones diferenciales. Enseñanza de las ciencias, 21(2), 265-280. Recuperado de: https://core.ac.uk/download/ pdf/13268099.pdf.
O’Donoghue, J. (2019). Una verdadera política nacional de inglés. Educación Futura. Recuperado de: http:// www.educacionfutura.org/una-verdadera-politicanacional-de-ingles/.

Ricouer, P. (1994). Ideología y utopía. Barcelona: Gedisa.

Robertson, C. (2021, 15 jul.). El aprendizaje del inglés y la educación como berramientas de prosperidad.

Searle, J. L. (1992). Intencionalidad. Un ensayo en la filosofía de la mente. Madrid: Tecnos, S.A.

Secretaría de Gobernación (2020). Programa Sectorial de Educación 2020-2024. Diario Oficial de la Federación.

SEMS-DGETA (2019). Informe de indicadores académicos. Texcoco, Estado de México.

SEP (2020). Programa de formación docente de educación media superior. Recuperado de: http: / / registro.desarrolloprofesionaldocente.sems.gob.mx/.

UnADM (2021). Presentan ILCE y UnADM convocatoria SEPA Inglés online 2021. Recuperado de: https:// www.unadmexico.mx/inicio-eventos/presentan-ilcey-unadm-convocatoria-sepa-ingles-online-2021.

Van Dijk, T. A. (1998). Ideología: una aproximación multidisciplinaria. Barcelona: Gedisa Editorial.

Vygotsky, L. S. (1981). Pensamiento y lenguaje. Buenos Aires: La Pléyade.

Zizek, S. (1999). El acoso de las fantasías. México: Siglo XXI.

Cómo citar este artículo:

Jaime Romero, B., Castillejos López, W., y Reyes Toxqui, Á. (2021). Intencionalidades y resistencias en el aprendizaje del inglés: referentes para diseñar estrategias didácticas efectivas. IE Revista de Investigación Educativa de la REDIECH, 12, e1013. doi: 10.33010/ie_rie_rediech.v12i0.1013. 